OPEN ACCESS

Edited by:

Clement Lafon Placette, Charles University, Czechia

Reviewed by:

Toni Gossmann,

Bielefeld University, Germany

Josselin Clo,

Charles University, Czechia

Catherine Rushworth,

University of Minnesota Twin Cities,

United States

${ }^{*}$ Correspondence:

Leonie C. Moyle

Imoyle@indiana.edu

Specialty section:

This article was submitted to Plant Development and EvoDevo,

a section of the journal

Frontiers in Plant Science

Received: 30 November 2020

Accepted: 01 March 2021

Published: 12 April 2021

Citation:

Moyle LC, Wu M and Gibson MJS (2021) Reproductive Proteins Evolve Faster Than Non-reproductive Proteins Among Solanum Species.

Front. Plant Sci. 12:635990.

doi: $10.3389 / f p / s .2021 .635990$

\section{Reproductive Proteins Evolve Faster Than Non-reproductive Proteins Among Solanum Species}

\author{
Leonie C. Moyle*, Meng Wu and Matthew J. S. Gibson \\ Department of Biology, Indiana University, Bloomington, IN, United States
}

Elevated rates of evolution in reproductive proteins are commonly observed in animal species, and are thought to be driven by the action of sexual selection and sexual conflict acting specifically on reproductive traits. Whether similar patterns are broadly observed in other biological groups is equivocal. Here, we examine patterns of protein divergence among wild tomato species (Solanum section Lycopersicon), to understand forces shaping the evolution of reproductive genes in this diverse, rapidly evolving plant clade. By comparing rates of molecular evolution among loci expressed in reproductive and non-reproductive tissues, our aims were to test if: (a) reproductive-specific loci evolve more rapidly, on average, than non-reproductive loci; (b) 'male'-specific loci evolve at different rates than 'female'-specific loci; (c) genes expressed exclusively in gametophytic (haploid) tissue evolve differently from genes expressed in sporophytic (diploid) tissue or in both tissue types; and (d) mating system variation (a potential proxy for the expected strength of sexual selection and/or sexual conflict) affects patterns of protein evolution. We observed elevated evolutionary rates in reproductive proteins. However, this pattern was most evident for female- rather than male-specific loci, both broadly and for individual loci inferred to be positively selected. These elevated rates might be facilitated by greater tissue-specificity of reproductive proteins, as faster rates were also associated with more narrow expression domains. In contrast, we found little evidence that evolutionary rates are consistently different in loci experiencing haploid selection (gametophytic-exclusive loci), or in lineages with quantitatively different mating systems. Overall while reproductive protein evolution is generally elevated in this diverse plant group, some specific patterns of evolution are more complex than those reported in other (largely animal) systems, and include a more prominent role for female-specific loci among adaptively evolving genes.

Keywords: female, male, molecular evolution, reproductive protein, sexual selection, Solanum, tomato

\section{INTRODUCTION}

The rapid evolution of reproductive proteins has been observed across many different animal species, including in groups as diverse as marine invertebrates, insects, and mammals (Swanson and Vacquier, 2002a; Clark et al., 2006; Turner and Hoekstra, 2008). This pattern is especially well established for proteins involved in male-specific functions, such as seminal fluid proteins, 
and sperm-egg and sperm-reproductive tract interactions. Although many factors can influence molecular evolutionary rates, two specific evolutionary forces-sexual selection and sexual conflict-have been proposed as primary drivers of this accelerated evolution, because both processes are expected to differentially affect reproductive traits and the proteins that underlie them. That is, traits mediating male-male competition and male-female mate choice experience unique selection to maximize mating and reproductive opportunities (Andersson, 1994), and often appear to evolve rapidly between species (Ritchie, 2007); therefore, the loci underpinning these reproductive traits might also be expected to display accelerated evolution and protein divergence between species (Swanson and Vacquier, 2002b; Clark et al., 2006; Vacquier and Swanson, 2011). This inference is supported by observations of especially exaggerated protein evolution in male-exclusive and male-biased loci (collated in Dapper and Wade, 2020), the sex that is usually subject to more intense sexual selection (Andersson, 1994). Importantly, if sexual selection or conflict are the most critical factors driving rapid reproductive protein evolution, this pattern should be observed in other groups of organisms that also experience these selective forces. Nonetheless, whether reproductive proteins routinely evolve rapidly in non-animal species remains unclear.

Among these other groups, flowering plants (angiosperms) have numerous reproductive traits that can influence the operation and intensity of intrasexual competition and/or mate choice (Lloyd and Webb, 1977; Willson, 1979; Stephenson and Bertin, 1983; Delph and Ashman, 2006; Moore and Pannell, 2011) - the two components of sexual selection. These include pollinator attraction traits, pollen competition traits, female reproductive tract ("pistil") traits (that influence pollen performance and fertilization success), and seed maturation traits (that can be used to exercise mate choice via selective abortion) (Willson, 1994; Delph and Havens, 1998; Skogsmyr and Lankinen, 2002). Accordingly, angiosperms may also be expected to exhibit elevated evolutionary rates in loci underpinning these traits, similar to those inferred in animals (Clark et al., 2006). Indeed, of the existing studies in angiosperms, some analyses have found evidence for faster protein evolution in reproductive loci (Szovenyi et al., 2013; Harrison et al., 2019), or greater representation of rapidly evolving genes among loci expressed in certain reproductive tissues (Gossmann et al., 2016). However, others have shown more equivocal patterns (e.g., Gossmann et al., 2014)-for example, sex-biased genes do not appear to evolve faster than non-sex-biased genes in several dioecious plant species (reviewed in Muyle, 2019)_or have inferred greater levels of constraint on reproductive-specific proteins (e.g., Darolti et al., 2018).

These more complex findings could reflect the influence of additional factors on rates of protein evolution in plants, including the opportunity for haploid selection and the complex effects of mating system variation. In the first case, plants often express a substantial proportion of their genome during the haploid phase of the life cycle (Hafidh et al., 2016), thereby exposing these 'gametophytic' loci to haploid selection. In flowering plants, gametophytic traits are largely reproductive and include 'male' functions such as pollen tube germination, growth, and interactions with the female pistil, and 'female' functions such as ovule signaling (Hafidh et al., 2016; Mizuta and Higashiyama, 2018). Compared to diploid-expressed loci, traits that rely on haploid gene function are expected to experience both stronger purifying selection against deleterious alleles, and elevated fixation rates (stronger positive selection) for advantageous alleles, because both deleterious and advantageous alleles will be visible to haploid selection regardless of dominance (Charlesworth and Charlesworth, 1992; Walsh and Charlesworth, 1992; Immler and Otto, 2018; Immler, 2019). Moreover, gametophytic-exclusive genes could also evolve differently from loci that are expressed in both gametophytic and sporophytic (diploid) tissue, if the latter also experience additional constraints or antagonistic effects because of their expression during both phases of the life cycle (Immler and Otto, 2018).

In addition to haploid selection, plants also often exhibit substantial variation in mating system, including among closely related species. This variation is predicted to have diverse effects on the nature, timing, and strength of selection, acting both on reproductive genes specifically, and across the genome more generally. In the specific case of reproductive genes, because the mating system affects the number of reproductive partners an individual experiences, it can directly influence the intensity of intrasexual competition, the opportunity for mate choice, and the magnitude of conflict over reproductive decisions (Delph and Havens, 1998; Skogsmyr and Lankinen, 2002; Brandvain and Haig, 2005; Clark et al., 2006; Mazer et al., 2010). Selection on traits affecting sexual competition and/or sexual conflict will be greatest in obligately outcrossing individuals, but weak or absent in obligately self-fertilizing species where the reproductive interests of both parents align perfectly (because they are the same individual). Even intermediate rates of self-fertilization can diminish the strength of sexual selection and thereby reduce, for example, the advantage of pollen competition traits (e.g., Mazer et al., 2018) and the spread of superior pollen-expressed genes (e.g., Peters and Weis, 2018). Mating system-specifically variation in the frequency of selfing-is also proposed to have more global effects on patterns of selection across the genome, regardless of whether genes have reproductive functions. Because selfing reduces effective population size $(\mathrm{Ne})$, the overall efficacy of selectionboth against deleterious alleles, and in favor of advantageous alleles-is expected to be reduced in selfing compared to outcrossing lineages (Charlesworth et al., 1993; Charlesworth and Wright, 2001; Mattila et al., 2018). Conversely, because selfing increases homozygosity, it can also reduce the genetic load in a population-by increasing the exposure of strongly deleterious recessive alleles to selection-as well as increase the early efficacy of selection on recessive advantageous alleles-by exposing them more rapidly to positive selection (Mattila et al., 2018). Together with specific effects on reproductive genes, these complex and sometimes antagonistic genome-wide effects might contribute to the heterogeneous inferences that have emerged from current studies of reproductive protein evolution in plants.

In this study, we examined genome-wide patterns and rates of protein evolution among four closely related species from 
the diverse plant genus Solanum. Our aims were to compare patterns of molecular evolution in loci expressed in different classes of tissue in order to evaluate whether: (a) reproductivespecific proteins evolve more rapidly, on average, than nonreproductive proteins; (b) 'male'-specific loci evolve at different rates than 'female'-specific loci; (c) genes expressed exclusively in gametophytic tissues evolve differently from genes expressed in sporophytic (diploid) tissue or in both tissue types; and (d) mating system variation (a potential proxy for the expected strength of sexual selection and/or sexual conflict) affects patterns of protein evolution. In addition, to help assess underlying causes of detected patterns, we also evaluated the influence of the magnitude and breadth of gene expression on observed rates of protein evolution. Our goals were to understand the forces shaping the molecular evolution of reproductive proteins in this plant group, and whether this differs from evolutionary patterns in other protein types and/or those proposed to shape reproductive protein evolution in animal systems.

\section{MATERIALS AND METHODS}

\section{Study Species}

Our analyses used gene expression and sequence data from each of four species within the wild tomato group (Solanum section Lycopersicum): S. lycopersicum (domesticated tomato; LA3475) and three wild relatives-S. pimpinellifolium (LA1589), S. pennellii (LA0716), and S. habrochaites (LA1777) (Supplementary Figure 1). (LA\#\#\#\# refers to the specific germplasm accession ID, as described in tgrc.ucdavis.edu, from which all or most of the data were obtained; Supplementary Table 1.) This group is a clade of 12 closely related diploid wild species along with the domesticated tomato, within the hyperdiverse $(\sim 1300$ species) plant genus Solanum (Sarkinen et al., 2013). All 12 wild tomato species arose within the last $\sim 2.5$ million years, consistent with recent rapid speciation in this clade (Pease et al., 2016a).

Mating system varies substantially across species in Solanum (Goldberg et al., 2010) and our four focal species differ in their mating system, most notably in whether species are historically self-incompatible (SI: S. pennellii and S. habrochaites) versus selfcompatible (SC: S. lycopersicum and S. pimpinellifolium). These species also have large estimated differences in their genetic effective population sizes $(\mathrm{Ne})$, that are consistent with these SI vs. SC mating system differences. For example, prior estimates of average heterozygosity from transcriptome-wide data are $0.28 \%$ and $0.245 \%$ for S. pennellii and S. habrochaites accessions respectively, and $0.04 \%$ for $S$. pimpinellifolium accessions (Supplementary Table 3 in Pease et al., 2016a). Heterozygosity is even lower in the domesticated tomato (S. lycopersicum) (e.g., Rick and Fobes, 1975). In Solanum, genetic self-incompatibility (the inferred ancestral state) is based on molecular interactions between proteins expressed in growing pollen tubes and pistilexpressed SRNase and other known proteins (Cruz-Garcia et al., 2003; Takayama and Isogai, 2005; Mcclure et al., 2011). SI is observed as arrest of pollen tube growth in the female reproductive tract (the pistil). Self-compatible lineages have lost function in one or more SI-mediating proteins (Stone, 2002), thereby allowing individuals to be fertilized by their own pollen, in addition to outcrossing. As with other angiosperms (Sicard and Lenhard, 2011), transitions to SC within Solanum are often followed by other morphological changes in floral size and structure, that further increase the frequency of self-pollination and reduce the number/diversity of mating partners (Rick, 1979). In our four focal species, SI species have larger flowers, longer reproductive tracts, and more pollen production on average, in comparison to SC species (Vosters et al., 2014), even though the transition to SC likely occurred within the last $\sim 0.5 \mathrm{MY}$ (the estimated age of the clade containing the two SC species here; Pease et al., 2016a). Both differences in the capacity to reject self pollen (i.e., SI versus SC), and these morphological differences, could have large effects on the average diversity of mating partners, and therefore the opportunity for and magnitude of intraspecific sexual selection and sexual conflict (Willson, 1994; Delph and Havens, 1998; Clark et al., 2006). In particular, while cross-pollination rates in SI species must be 100\%, these have been estimated to range from 0 to $40 \%$ in wild SC species, including S. pimpinellifolium (e.g., Rick et al., 1977, 1978).

\section{Quantifying Gene Expression and Determining Tissue-Specificity}

Our analyses used gene expression (RNAseq) data collected individually from leaf (up to five developmental stages), root, seed, vegetative meristem, stem, flower (several stages), style, pollen, and ovule tissues in each accession. RNAseq data were obtained from seven publically available SRA projects (Supplementary Table 1), mostly drawn from three previously published analyses (Koenig et al., 2013; Ichihashi et al., 2014; Pease et al., 2016b), except data from ovule tissue which has not been previously published. Note that style and pollen data were not available specifically for S. pimpinellifolium. Sources of each dataset are described in detail in Supplementary Table 1. Procedures for generating the ovule RNAseq data are described in the Supplementary Text. Each tissue had 1-6 replicates (generally 3) per accession (Supplementary Table 1).

For each library within each tissue, we processed the raw reads by filtering adapter sequences and low quality bases with TRIMMOMATIC (Bolger et al., 2014). Reads were then mapped against the tomato reference genome (ITAG 2.4) using STAR (Dobin et al., 2013), with default settings excluding alignments with $>10$ mismatches. Numbers of reads mapped onto genic regions were estimated with FEATURECOUNTS (Liao et al., 2014). We normalized the read counts from each library by calculating TPM (transcripts per million) and then calculated the mean normalized read counts for each tissue of each species.

We first classified loci into three general classes of genereproductive (RP), vegetative (VG), and general (GR) (i.e., expressed in both reproductive and non-reproductive/vegetative tissue types). Reproductive genes were required to be expressed $(\mathrm{TPM}>2)$ in at least one reproductive tissue (i.e., style, pollen, ovule), and to have no or trace expression (TPM $<0.5)$ in any of the remaining tissues (i.e., leaf, root, stem, seed, root, vegetative meristem), in at least two of the three investigated species for 
which we had all tissues available (i.e., S. lycopersicum, S. pennellii, and S. habrochaites). (Because S. pimpinellifolium lacked some of the tissue types in our RNA-seq data, we did not include this species in the filtering/determination of tissue-specificity). Vegetative genes were defined similarly by requiring expression in at least one vegetative (non-reproductive) tissue, and no/trace expression in any reproductive tissue. Generally expressed (GR) genes were expressed in at least one reproductive tissue and also in at least one vegetative tissue.

Using similar criteria, we also identified a set of tissue-specific genes, defined as those only expressed in one specific tissue $(\mathrm{TPM}>2)$ and not in any other tissue $(\mathrm{TPM}<0.5)$ in at least two out of the three species for which we had all tissue types (i.e., S. lycopersicum, S. pennellii, and S. habrochaites). For these analyses, we focused on specific tissues (i.e., leaf, root, stem, seed, vegetative meristem, style, pollen, ovule) for which we had sufficient sampling for at least three species (Supplementary Table 1). Finally, using the same criteria and the expression domains in the three species for which we had all tissues represented, we also classified transcripts as gametophytic-exclusive (pollen and/or ovule expression only) versus sporophytic-exclusive versus both gametophytic and sporophytic (pollen and/or ovule expression, and at least one other diploid tissue), as well as ovule-specific versus expressed in both ovules and at least one sporophytic (diploid) tissue, and pollen-specific versus expressed in both pollen and at least one sporophytic (diploid) tissue.

\section{Estimating Rates of Protein Evolution}

As with prior studies (Clark et al., 2006 and references therein), we used $\mathrm{dN} / \mathrm{dS}$ - the ratio of the number of nonsynonymous substitutions per non-synonymous site to the number of synonymous substitutions per synonymous site-as our estimate of protein evolution. Although $\mathrm{dN} / \mathrm{dS}$ is usually $<1$-because most functional loci experience some constraint on non-synonymous changes-larger values of $\mathrm{dN} / \mathrm{dS}$ are consistent with faster protein evolution. Note that estimates of $\mathrm{dN} / \mathrm{dS}$ can be influenced by intraspecific polymorphism when it is high compared to fixed differences between species (see Hahn, 2018, Chapter 7). However, estimates of heterozygosity within each of our wild accessions are small compared to divergence (e.g., dS) estimates across these species (Supplementary Text), indicating that most of the SNPs contributing to our estimates of $\mathrm{dS}, \mathrm{dN}$, and $\mathrm{dN} / \mathrm{dS}$, are fixed differences.

We estimated $\mathrm{dN} / \mathrm{dS}$ for each locus in our dataset, to calculate mean rates of protein evolution in different classes of genes, and to identify the set of loci with estimated $\mathrm{dN} / \mathrm{dS}>1-$ a pattern consistent with positive selection for protein evolution at that locus. In addition, to compare the overall $\mathrm{dN} / \mathrm{dS}$ for loci on SI versus SC branches, we compared estimates from sequences that were concatenated across groups of genes (described below). Note that low transcript coverage reduces the power to accurately call SNPs at heterozygous sites and can thereby introduce errors into inferred sequences and statistical estimates based on these; this can be a concern in low coverage RNA-seq data. However, the average per locus raw read count in our dataset ranges from $\sim 160$ to $>800$ (Supplementary Table 2), indicating that we have ample power to accurately call SNPs with these transcript data.

To generate the sequence alignments for these analyses, we combined the RNA-seq data used for quantifying gene expression (above) with whole-transcriptome data from the same four accessions previously generated in Pease et al. (2016a), and with publicly available DNA-seq data (Supplementary Table 3); the latter datasets allowed us to augment our RNAseq data with additional sequences for loci whose expression was not detected for one or more of our four species. These reads were aligned against the tomato reference genome by STAR, with defaults excluding alignments with $>10$ mismatches, as with the previous reference-aligned single copy ortholog dataset (Pease et al., 2016a). The aligned data were processed using SAMTOOLS followed by MVFTOOLS to generate the sequence alignments of orthologous genes. We only retained transcript sequence alignments longer than 200 bps after removing gaps and indels, leaving us with 21,216 sequence alignments (loci) for downstream analyses.

For each locus, we used PAML (Yang, 2007) to estimate $\mathrm{dN} / \mathrm{dS}$ across the branches of a given phylogenetic topology among the four investigated species, using the input species tree [(lycopersicum, pimpinellifolium), (pennellii, habrochaites)] which reflects the known species topology (Supplementary Figure 1). To do so, we used the one-ratio (M0, or model $=0$ ) codeml model in PAML, that generates a single $\mathrm{dN} / \mathrm{dS}$ estimate across all branches for each locus (that is, $\mathrm{dN} / \mathrm{dS}$ is fixed at one ratio for each gene). Outputs from this model were used to compare the mean and distribution of $\mathrm{dN} / \mathrm{dS}$ for genes among three broad categories [reproductive (RP), vegetative (VE), and general (GR)], as well as between each of six tissue-specific groups classified by their expression in only a single tissue (pollen, style, ovule, leaf, root, or stem; vegetative meristem and seed were excluded from the latter analyses because they had no exclusively tissue-specific genes), and between loci that had gametophytic versus sporophytic, or gametophytic + sporophytic, domains of gene expression. We also compared loci expressed in ovules only versus expressed in ovules and at least one sporophytic (diploid) tissue, or in pollen only versus expressed in pollen and at least one sporophytic tissue.

Second, in addition to locus-by-locus dN/dS comparisons, we also evaluated overall $\mathrm{dN} / \mathrm{dS}$ estimates along SC branches in comparison to those along SI branches. Among our four species, the two self-compatible species (S. lycopersicum and S. pimpinellifolium) are most closely related to each other, and the lengths of SC branches (those leading to S. lycopersicum and $S$. pimpinellifolium) are much shorter than the length of SI branches (those leading to S. pennellii and S. habrochaites) (Supplementary Figure 1). As a result, branch lengths (especially for SC lineages) were too short to reliably estimate lineagespecific dN/dS for each locus individually. Therefore, for these analyses we concatenated all loci within each class of genes to be compared, and then estimated $\mathrm{dN} / \mathrm{dS}$ across the concatenated set of loci for SI and SC branches separately. For example, for reproductive (RP) loci, we concatenated all RP loci and estimated $\mathrm{dN} / \mathrm{dS}$ across the entire concatenated set, allowing for different $\mathrm{dN} / \mathrm{dS}$ estimates (variable rates) on SI versus SC branches. 
Because of the small numbers of loci concatenated for some of our individual tissues, we limited SI vs. SC branch-specific estimates to our three broad classes of loci (RP, VG, and GR) only.

Because the phylogeny of our species contains one internal branch, for these analyses we used two alternative multi-rate (model $=2$ ) codeml models in PAML. For the first, threeratio, model we allowed the internal branch to have a rate that differed from the tip branches leading either to SC species, or to SI species. For the second, two-ratio, model, we classified the internal branch as 'SI' (i.e., dN/dS was estimated separately for tip branches leading to SC species, versus a background rate for the tip branches leading to SI species plus the internal branch). This approach was used to estimate branch-specific rates (dN/dS estimates) for SI versus SC branches for each of our three general classes of loci (RP, VG, and GR). Note we report three-ratio results in the main text, and two-ratio results in the supplement.

\section{Comparing Protein Evolution Rates}

All statistical analyses were performed in $\mathrm{R}$ version 3.6.3 ( $\mathrm{R}$ Core Team, 2015). For all comparisons, we first removed genes showing unusually high values $(\mathrm{dN} / \mathrm{dS}>10)$ from the PAML output, as these were due to estimation limitations within PAML (e.g., a lack of synonymous substitutions across the branch(es) used for estimation) or otherwise from poor sequence quality or alignment.

To compare the mean rate of per locus protein evolution $(\mathrm{dN} / \mathrm{dS})$ between different classes of loci, we fit generalized linear models (GLMs). Shapiro-Wilk tests of normality and quantilequantile plots were used to assess normality in the distribution of $\mathrm{dN} / \mathrm{dS}$; in all categories, $\mathrm{dN} / \mathrm{dS}$ was heavily skewed toward lower values and zero-inflated (reflecting the fact that most expressed genes are under purifying selection). To accommodate this in our models, we assume a gamma residual distribution with an identity link function, rather than the standard Gaussian assumption, after confirming the suitability of this distribution with maximum likelihood (using the fitdistrplus $\mathrm{R}$ package). Following GLMs, to compare the mean estimated $\mathrm{dN} / \mathrm{dS}$ between specific classes of loci, we made pairwise comparisons using Tukey post hoc tests, adjusting $p$-values for multiple comparisons. Specifically, post hoc test $p$-values were adjusted using the default 'single-step' method in the R package 'multcomp' (Hothorn et al., 2008), which adjusts $p$-values based on the joint normal or $t$ distribution of the linear function. In addition, we compared the proportion of positively selected genes (i.e., $\mathrm{dN} / \mathrm{dS}>1$ ) between different classes of loci by performing Chi-Squared tests of independence and, for specific tests of enrichment in single categories (e.g., in RP relative to GR), one-sided Fisher's exact tests.

To assess evidence for differences in $\mathrm{dN} / \mathrm{dS}$ between mating systems, we aimed to compare $\mathrm{dN} / \mathrm{dS}$ estimates from concatenated data, as calculated separately for SI and SC branches. Both biological and technical factors make these comparisons challenging to interpret. Biologically, overall differences in $\mathrm{Ne}$ and therefore in the expected efficacy of positive and purifying selection, could generate global differences in rates of protein evolution between SC and SI lineages (see the section
"Introduction"), that are unrelated to their potential differences in the historical strength of sexual selection. Technically, in our specific dataset, the two SC species are much more closely related than the two SI species, so there are large differences in branch lengths (number of substitutions) over which we are estimating dN/dS values for SI versus SC branches. Therefore, to assess whether exclusively reproductive (RP) loci had a different pattern of molecular evolution on SI versus SC branches in comparison to other loci, we compared the observed difference in $\mathrm{dN} / \mathrm{dS}$ between SI and SC branches for RP genes to an estimate of the 'baseline' genome-wide difference in $\mathrm{dN} / \mathrm{dS}$ between $\mathrm{SI}$ and SC branches. This baseline was described by the SI vs. SC difference in our set of generally expressed genes (GR), which is expected to be due to factors unrelated to variation in sexual selection. Because, we have only a single estimate (from the concatenated data) for each class of gene for each branch type, standard parametric tests can't be used. Instead, we used bootstrap resampling to estimate the variance around the mean SI vs. SC difference in $\mathrm{dN} / \mathrm{dS}$ in the GR dataset, and then to evaluate whether the observed SI vs. SC difference in RP genes falls outside the range of this variance (i.e., is larger or smaller than the $95 \%$ CI of this distribution). For each bootstrap replicate ( $N=1000$ replicates), we re-sampled 500 loci at random from the GR dataset, estimated $\mathrm{dN} / \mathrm{dS}$ for SI and SC branches and the difference between these two estimates. Our observed $\mathrm{dN} / \mathrm{dS}$ difference (SC-SI rate) was compared to the resulting distribution of 1000 replicate $\mathrm{dN} / \mathrm{dS}$ differences from GR loci.

Finally, to confirm that detected differences in $\mathrm{dN} / \mathrm{dS}$ values between classes of genes were not driven by differences in rates of synonymous mutation-which, for example, can systematically vary according to genomic location or nucleotide content-we also evaluated differences in $\mathrm{dN}$ and $\mathrm{dS}$ separately for these classes of genes (Supplementary Text).

\section{Assessing the Effect of Gene Expression Level on Molecular Evolutionary Rates}

Numerous studies have shown that rates of protein evolution can be influenced by the magnitude and breadth of gene expression, such that highly expressed genes and/or genes expressed in a broader number of tissues tend to have systematically lower estimated dN/dS (Meisel, 2011). To assess the effect of the magnitude and breadth of gene expression on our inferences, we also compared average $\mathrm{dN} / \mathrm{dS}$ values between genes expressed in different numbers of tissues [ranging from 1 (tissue-specific loci) through to 8 (expressed in all tissues examined)], using the criteria that a gene be expressed $>2$ TPM in a specific tissue in all species for which we had data on that tissue-type. Within each of the broad classes of RP, VG, and GR loci, we also assessed the quantitative relationship between mean gene expression level (averaged across all tissues in which a locus was expressed) and estimated $\mathrm{dN} / \mathrm{dS}$. For this analysis, we constructed a multiple regression model of $\mathrm{dN} / \mathrm{dS}$ (square root transformed to approximate normality), including both mean gene expression (TPM), tissue class (RP, VG, or GR), and their interaction term as independent variables. 


\section{Assessing Enrichment of Functional Categories Among Adaptively Evolving Loci}

To test for functional enrichment among our loci with $\mathrm{dN} / \mathrm{dS}>1$, we performed a Gene Ontology (GO) term enrichment analysis using Panther version 14 (Ashburner et al., 2000; Mi et al., 2019; The Gene Ontology Consortium, 2019). We performed separate tests for GR, VE, and RP tissue categories, as these classes had large sample sizes appropriate for tests of enrichment. Loci were evaluated using both the biological process and molecular function annotation data sets, and significance was determined using Fisher's Exact tests with false discovery rate correction. False discovery rates were calculated using the BenjaminiHochberg procedure, using a critical value of 0.05 to filter results. All statistics were calculated using the automated PANTHER Overrepresentation Tests.

\section{RESULTS}

\section{Reproductive Genes Evolve at Modestly Faster Rates, and Have Proportionally More Genes Under Positive Selection}

Reproductive (RP) genes had an elevated mean rate of protein evolution compared to loci expressed in both reproductive and non-reproductive (general/GR) tissues (Post hoc $Z=2.548$, $p=0.0108$ ) (Figure 1, Table 1, and Supplementary Tables 4, 5). The mean $\mathrm{dN} / \mathrm{dS}$ for vegetative-specific (VG) loci fell between

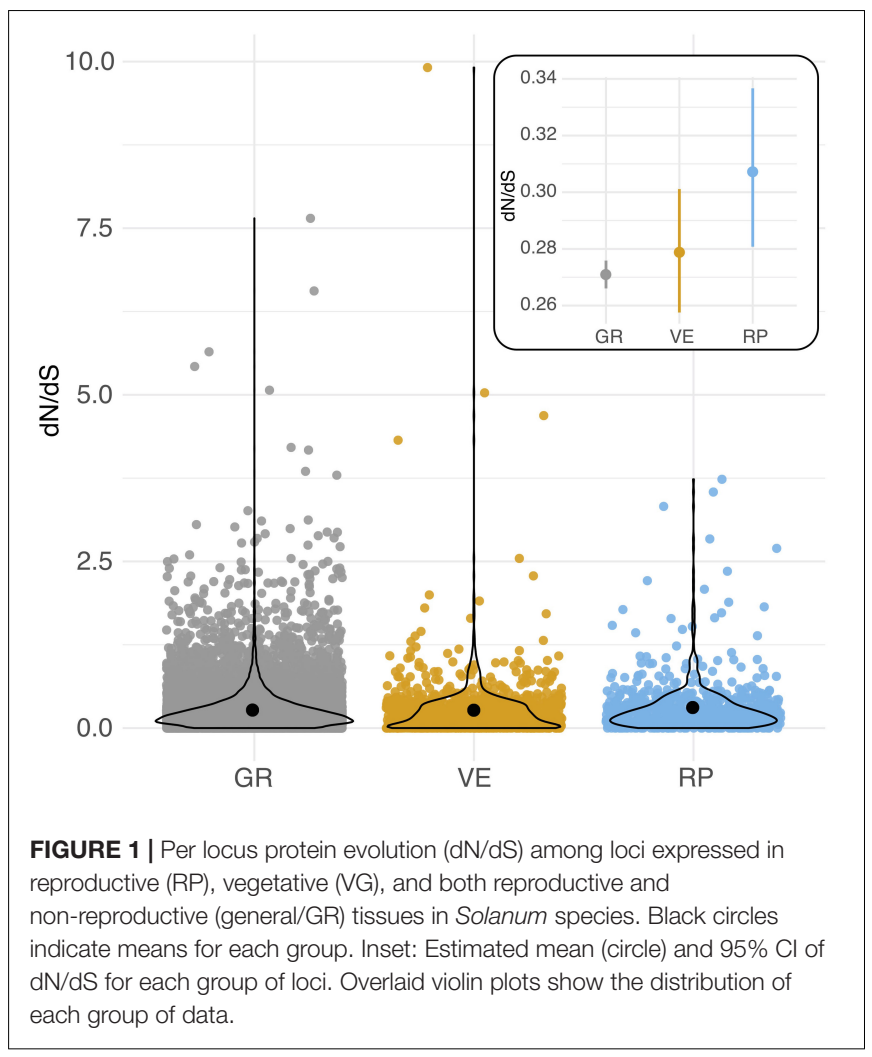

these two other classes of genes and did not differ from either of them (Table 1). These observed differences were not driven by variation in synonymous mutation rates $(\mathrm{dS})$ between these classes of genes (Supplementary Text 3, Supplementary Figure 2, and Supplementary Table 6). In addition, the proportion of genes with $\mathrm{dN} / \mathrm{dS}>1$ differed by group (Table $\mathbf{1}$ ). A significantly greater proportion of reproductive loci (28 of 670 , or $\sim 4.17 \%$ ) are inferred to be evolving adaptively compared to general loci $(2.45 \%)$ (Fisher's exact test, $p=0.0067$; Table 1 and Supplementary Table 5). The proportion of vegetative loci evolving adaptively (2.81\%) was marginally lower than reproductive loci (Fisher's exact test, $p=0.093$ ), and did not differ from general loci (Table 1).

\section{Female- Rather Than Male-Specific Genes Evolve the Most Rapidly}

Comparisons among tissue-specific genes indicated no evidence that pollen-specific loci evolve more rapidly than loci from female-derived tissues (style and ovule) (Figure 2, Table 2, and Supplementary Table 7). Instead, style- and ovule-specific loci had the highest mean $\mathrm{dN} / \mathrm{dS}$ values of all tissue groups, and post hoc pairwise tests indicated that style mean rate was significantly greater than rates in root-specific and leafspecific loci, and ovule mean rate was significantly greater than leaf mean rate (Figure 2, Table 2, and Supplementary Table 8). In comparison, pollen loci had a mean rate that was intermediate between these groups of tissue-specific genes, and statistically indistinguishable from each other tissue (Supplementary Table 8). Note that many fewer loci were available for tissue-specific comparisons, especially leaf- and stem-exclusive loci (Table 2), and two tissues-vegetative meristem and seed-had no detected tissue-exclusive loci.

Similar to comparisons of mean rates, the proportion of genes with $\mathrm{dN} / \mathrm{dS}>1$ differed by tissue (Table 2). Moreover, this proportion was significantly higher in style tissue-where 6 of 25 loci were inferred to be evolving adaptively-compared to all other tissues (Fisher's exact test, $p=5.046 \times 10^{-5}$; Table 2). For ovule-exclusive loci, the proportion adaptive evolving loci ( 3 of 71) was also greater than in all other tissues (excluding style-specific loci) (Fisher's exact test, $p=0.0376$ ) (Table 2).

\section{Gametophytic-Exclusive Proteins Do Not Evolve More Rapidly Than Sporophytic-Exclusive Proteins, or Proteins Expressed in Both Tissue Types}

Although the mean rate of protein evolution in gametophytic genes was slightly higher than the rate of protein evolution in sporophytic-exclusive loci, this difference was not significant $(t=-0.452, P=0.588$ ) (Table 3 and Supplementary Table 9). Gametophytic-exclusive proteins also do not evolve faster than those expressed in at least one gametophytic and one sporophytic tissue $(t=-1.498, P=0.134$ ) (Table 3$)$. Similar to mean rate comparisons, the proportion of gametophytic loci with $\mathrm{dN} / \mathrm{dS}>1$ (3 of 86 , or $3.49 \%$ ) did not differ from this proportion in sporophytic-exclusive genes (68 of 2033, or 
TABLE 1 | Rates of protein evolution (dN/dS) in different broad classes of loci.

\begin{tabular}{|c|c|c|c|c|c|c|c|c|}
\hline \multirow[b]{2}{*}{ Class } & \multicolumn{5}{|c|}{ Per locus dN/dS } & \multicolumn{3}{|c|}{ Loci with dN/dS > 1} \\
\hline & N loci & Mean & SE & Median & Tukey test ${ }^{1}$ & Number & Proportion ${ }^{2}$ & Fisher's exact tests ${ }^{1}$ \\
\hline General & 16221 & 0.2709 & 0.0026 & 0.20 & $a$ & 399 & 0.0246 & $\mathrm{a}$ \\
\hline Vegetative & 923 & 0.2783 & 0.0111 & 0.19 & $a b$ & 26 & 0.0282 & $a b$ \\
\hline Reproductive & 670 & 0.3071 & 0.0142 & 0.21 & $b$ & 28 & 0.0418 & $b$ \\
\hline
\end{tabular}

dN/dS calculated for each locus using a one ratio test in PAML.

${ }^{1}$ See Supplementary Table 4.

${ }^{2}$ The proportion of genes with $d N / d S>1$ differed among classes of loci $\left(X^{2}=7.9696, p=0.0186\right)$.

TABLE 2 | Rates of protein evolution (dN/dS) in tissue-specific loci.

\begin{tabular}{|c|c|c|c|c|c|c|c|}
\hline \multirow[b]{2}{*}{ Group } & \multicolumn{4}{|c|}{ Per locus dN/dS } & \multicolumn{3}{|c|}{ Loci with dN/dS > 1} \\
\hline & $N$ & Mean & SE & Median & Tukey test ${ }^{1}$ & Number & Proportion $^{2}$ \\
\hline Leaf & 3 & 0.0978 & 0.0657 & 0.00 & a & 0 & 0 \\
\hline Stem & 2 & 0.1925 & 0.1714 & 0.19 & abc & 0 & 0 \\
\hline Root & 124 & 0.2103 & 0.0693 & 0.19 & $\mathrm{ab}$ & 0 & 0 \\
\hline Pollen & 13 & 0.2521 & 0.1046 & 0.16 & $a b c$ & 0 & 0 \\
\hline Ovule & 71 & 0.3418 & 0.0809 & 0.25 & bc & 3 & 0.042 \\
\hline Style & 25 & 0.6668 & 0.1685 & 0.17 & C & 6 & 0.240 \\
\hline
\end{tabular}

${ }^{1}$ See Supplementary Table 8.

${ }^{2}$ The proportion of genes with $d N / d S>1$ differed by tissue $X^{2}=33.707$, $\left.p=2.72 \times 10^{-6}\right)$. One-sided Fisher's exact tests indicated this proportion was greater in style compared to all other tissues $\left(p=5.046 \times 10^{-5}\right)$, and greater in ovule compared to all other tissues except style ( $p=0.03756)$.

3.34\%), and was slightly but not statistically larger than this proportion in gametophytic + sporophytic genes (383 of 15778, or $2.43 \%$ ) (Table 3 ).

As observed in the general gametophytic comparisons, mean rate of protein evolution in ovule-exclusive loci was slightly higher than the rate of protein evolution in loci expressed in ovules and at least one sporophytic tissue (Table 3), but this difference was not significant (GLM $t=1.212, p=0.225$; Supplementary Table 9). The proportion of ovule-exclusive loci with $\mathrm{dN} / \mathrm{dS}>1$ (3 of 71 , or $4.23 \%$ ) also did not differ from the proportion of ovule + sporophytic loci with $\mathrm{dN} / \mathrm{dS}>1$ (225 of 7884 , or $2.85 \%$ ) (Table 3 ). Neither the mean rate of protein evolution (GLM $t=-0.037, p=0.971$; Supplementary Table 9) nor the proportion of loci with $\mathrm{dN} / \mathrm{dS}>1$ (Table 3 ) differed between pollen-exclusive loci and loci expressed in pollen and at least one sporophytic tissue.

\section{Patterns of Protein Evolution Show Mating-System Effects That Are Not Specific to Reproductive Loci}

Consistent with genome-wide effects of lower $\mathrm{Ne}$ in our self-compatible lineages, rates of protein evolution were inferred to be higher overall on self-compatible branches in both GR and RP classes of loci, although not for VG loci (Table 4); in the latter case, VG rates were numerically higher on SI branches. Results were similar for two-ratio models (Supplementary Table 10). The observed difference in rates between SC and SI branch for RP loci (0.0338) did not fall outside the $95 \%$ CI of bootstrapped values (0.0007-0.0797) from the broader class of GR genes; instead 33\% of bootstrapped samples had a $\mathrm{dN} / \mathrm{dS}$ difference smaller than observed for RP loci.

\section{Higher Rates of Protein Evolution Are Consistently Associated With Greater Tissue-Specificity}

Across loci classified according to the number of tissues in which they were expressed at $>2$ TPM (from 1 to 8 tissues), mean $\mathrm{dN} / \mathrm{dS}$ was highest in loci expressed in a single tissue and consistently declined across loci expressed in increasingly more tissues (Figure 3, Table 5, and Supplementary Tables 11, 12). Similarly, both mean expression level and tissue class (GR, $\mathrm{RP}, \mathrm{VG}$ ) affected the per locus estimates of protein evolution $(\mathrm{dN} / \mathrm{dS})$, as did their interaction (Table 6), indicating that the relationship between expression level and rates of protein

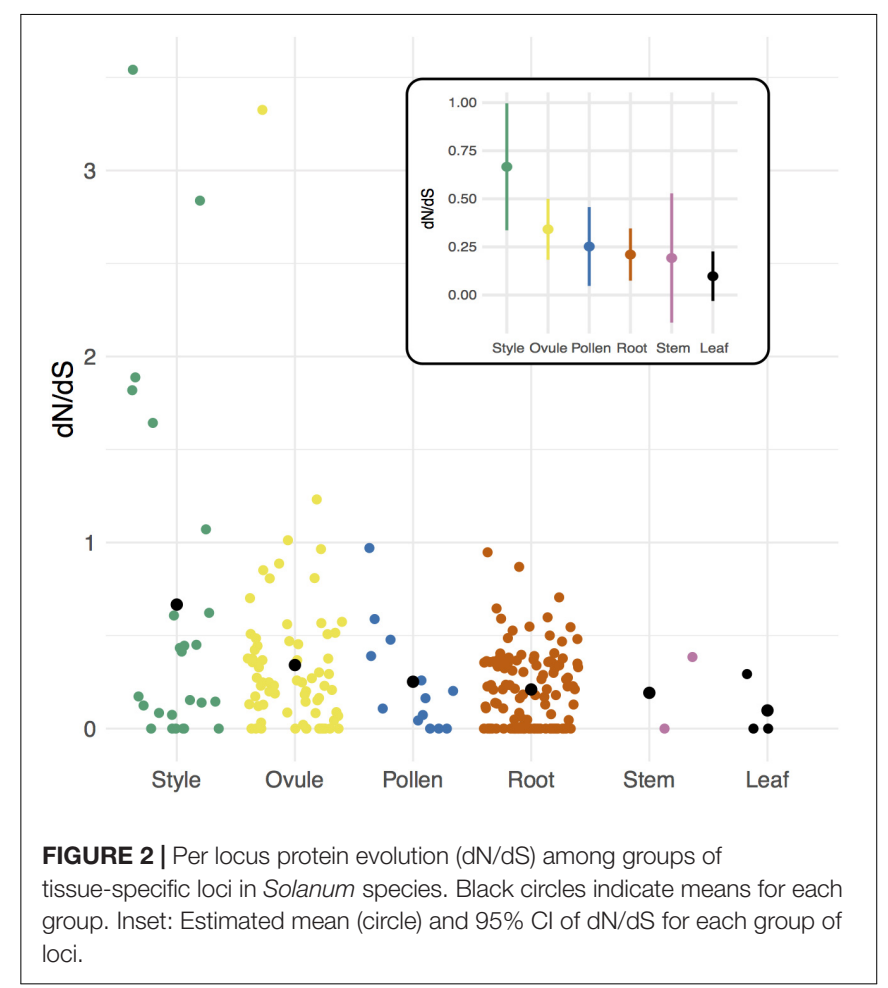


TABLE 3 | Rates of protein evolution (dN/dS) in gametophytic (GM) versus sporophytic (SP) or gametophytic + sporophytic (GM + SP) loci.

\begin{tabular}{|c|c|c|c|c|c|c|c|}
\hline \multirow[b]{2}{*}{ Group } & \multicolumn{3}{|c|}{$\mathrm{dN} / \mathrm{dS}$} & \multicolumn{4}{|c|}{ Loci with $\mathrm{dN} / \mathrm{dS}>1$} \\
\hline & $N$ & Mean & SE & Median & Number & Proportion & $p^{1}$ \\
\hline \multicolumn{8}{|c|}{ a) Gametophytic vs. sporophytic } \\
\hline Gametophytic only & 86 & 0.3275 & 0.0576 & 0.24 & 3 & 0.0349 & 0.5574 \\
\hline Sporophytic only & 2033 & 0.2958 & 0.0586 & 0.21 & 68 & 0.0334 & \\
\hline \multicolumn{8}{|l|}{ b) Gametophytic vs. GM + SP } \\
\hline Gametophytic only & 86 & 0.3275 & 0.0393 & 0.24 & 3 & 0.0349 & 0.2833 \\
\hline Gametophytic + sporophytic & 15778 & 0.2685 & 0.0394 & 0.20 & 383 & 0.0243 & \\
\hline \multicolumn{8}{|l|}{ c) Ovule loci } \\
\hline Ovule only & 71 & 0.3418 & 0.0036 & 0.25 & 3 & 0.0423 & 0.8542 \\
\hline Ovule + sporophytic & 7884 & 0.2874 & 0.0448 & 0.21 & 225 & 0.0285 & \\
\hline \multicolumn{8}{|l|}{ d) Pollen loci } \\
\hline Pollen only & 13 & 0.2521 & 0.0679 & 0.16 & 0 & 0.0000 & 0.8316 \\
\hline Pollen + sporophytic & 277 & 0.2496 & 0.0695 & 0.18 & 4 & 0.0144 & \\
\hline
\end{tabular}

Within each GLM, tissue groups did not differ in mean dN/dS (Supplementary Table 9).

${ }^{1}$ Fisher's exact tests.

TABLE 4 | Estimates of dN/dS for SC and SI branches separately, for loci concatenated within each broad class of genes.

\begin{tabular}{|c|c|c|c|c|c|c|c|c|c|c|}
\hline Class & \# genes & \# codons & Null model & $\begin{array}{c}\text { Variable rate } \\
\text { model }\end{array}$ & 2*LnL & $p$-value & $\begin{array}{c}\text { Sl-branch } \\
\text { dN/dS }\end{array}$ & $\begin{array}{c}\text { SC-branch } \\
\text { dN/dS }\end{array}$ & $\begin{array}{l}\text { Internal } \\
\text { Branch }\end{array}$ & SC-SI diff. \\
\hline GR & 16221 & 6619178 & -28576265.18 & -28575900.38 & 729.61282 & $<1 \times 10 x-16$ & 0.2224 & 0.2617 & 0.1901 & 0.0392 \\
\hline $\mathrm{RP}$ & 904 & 110171 & -478937.9344 & -478932.4222 & 11.024336 & 0.00404 & 0.2285 & 0.2623 & 0.1999 & 0.0338 \\
\hline VG & 1300 & 78355 & -344551.0442 & -344546.389 & 9.310318 & 0.00951 & 0.2267 & 0.2042 & 0.1850 & -0.0225 \\
\hline
\end{tabular}

Results are from 3 ratio model in PAML, with input species tree: [(Slyc \#1, Spim \#1), (Spen \#2, Shab \#2)].

evolution varies depending upon the broad tissue class. In particular, while the relationship between mean expression level and $\mathrm{dN} / \mathrm{dS}$ was significantly negative for generally expressed (GR) loci, the 95\% CI for this slope overlapped zero for $\mathrm{RP}$ loci, indicating little evidence for a relationship between expression level and rate of protein evolution for the RP class of loci (Supplementary Figure 3 and Supplementary Table 13). Classes of genes also differed in their overall mean gene expression (mean total TPM), which was highest in RP genes $(505.2 \pm 23.7)$ compared to GR genes $(398.7 \pm 4.2)$, and VG loci (43.9 \pm 4.5 ) (Supplementary Table 14). Finally, mean gene expression level also differed between some tissue-specific loci, with style and ovule loci having the highest average gene expression (Supplementary Table 15).

These findings are generally consistent with greater evolutionary constraint on loci with broader expression domains, across all our loci. In addition, for genes expressed in at least one reproductive and non-reproductive tissue (i.e., general loci), they indicate greater constraint on genes with higher magnitudes of gene expression.

\section{Adaptively Evolving Reproductive Proteins Are Not Enriched for Specific Functional Categories, but Do Include Roles in Sexual Interactions}

We found no evidence for enrichment of functional gene ontology categories among loci for which we found $d \mathrm{~N} / \mathrm{dS}>1$, including among reproductive loci inferred to be adaptively evolving (data not shown). Nonetheless, among these reproductive loci, we did identify several genes with clear functional roles in sexual interactions (Supplementary Table 16).

\section{DISCUSSION}

Reproductive proteins appear to evolve rapidly in many animal groups. Here, we evaluated evidence for elevated reproductive protein evolution in four closely related flowering plant species, along with the potential influence of three factors on observed patterns: variation in mating system (and therefore the possible strength of sexual selection), in gametophytic gene expression (and therefore the strength of haploid selection), and in the breadth and/or magnitude of gene expression. We found evidence for elevated rates of reproductive protein evolution, globally across the genome and in some specific reproductive tissues. Moreover, these elevated rates appear to be more characteristic of female- rather than male-specific loci. Among the three broad factors that might influence these patterns, we found that elevated evolution was consistently associated with more narrow domains of gene expression, but not with expression in haploid versus diploid tissues; evolutionary rate differences between lineages with different mating systems (self-compatible versus self-incompatible) were more complex and not associated with specific shifts in 


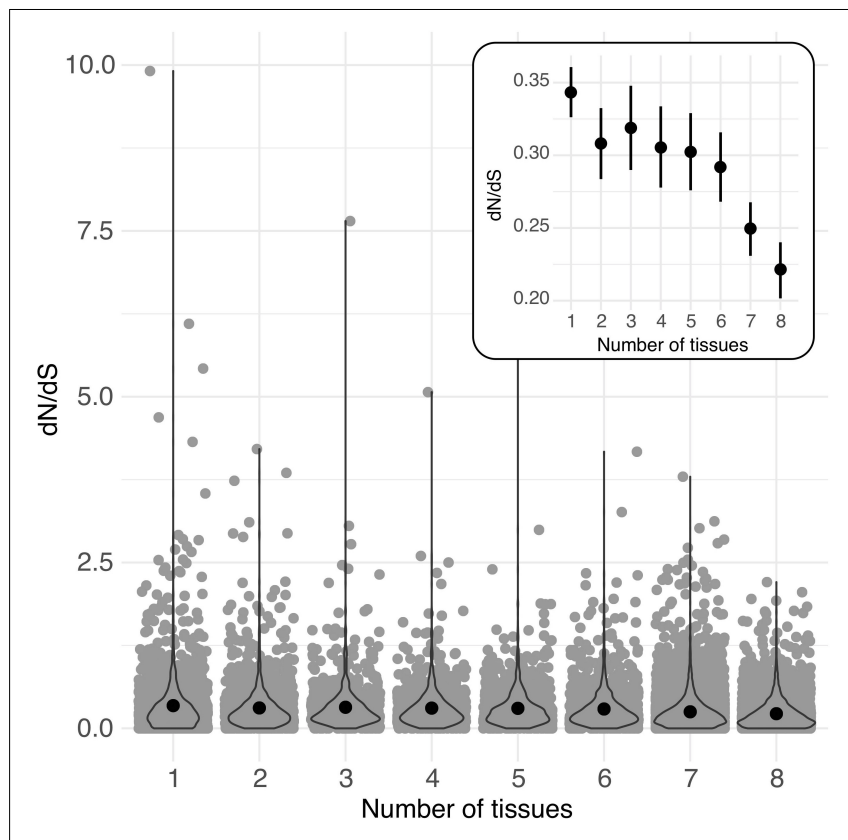

FIGURE 3 | Per locus protein evolution (dN/dS) among groups of loci expressed (>2 TPM) in different numbers of tissues (from 1 to 8 ) in Solanum species. Black circles indicate means for each group. Inset: Estimated mean (circle) and $95 \% \mathrm{Cl}$ of dN/dS for each group of loci. Overlaid violin plots show the distribution of each group of data.

TABLE 5 | Rates of protein evolution (dN/dS) in loci according to breadth of tissue expression.

\begin{tabular}{lccccc}
\hline $\begin{array}{l}\text { \# Tissues } \\
\text { expressed in }\end{array}$ & N loci & $\begin{array}{c}\text { Mean sqrt } \\
\text { (dN/dS) }\end{array}$ & SE & Median & $\begin{array}{c}\text { Post hoc } \\
\text { Tukey test }\end{array}$ \\
\hline 1 & 1892 & 0.3429 & 0.0088 & 0.24 & $\mathrm{~d}$ \\
2 & 1528 & 0.3080 & 0.0125 & 0.22 & $\mathrm{~cd}$ \\
3 & 901 & 0.3183 & 0.0148 & 0.23 & $\mathrm{~cd}$ \\
4 & 930 & 0.3053 & 0.0142 & 0.23 & $\mathrm{~cd}$ \\
5 & 1083 & 0.3022 & 0.0135 & 0.24 & $\mathrm{C}$ \\
6 & 1507 & 0.2919 & 0.0122 & 0.21 & $\mathrm{C}$ \\
7 & 7206 & 0.2498 & 0.0094 & 0.19 & $\mathrm{~b}$ \\
8 & 3173 & 0.2213 & 0.0098 & 0.16 & $\mathrm{a}$ \\
\hline
\end{tabular}

${ }^{1}$ See Supplementary Table 12.

TABLE 6 | Relationship between mean gene expression level and estimated dN/dS, across loci in each broad class (GR, RP, VG) of loci (linear model).

\begin{tabular}{lcrrrr}
\hline & DF & SS & MS & F & Pr $>$ F \\
\hline Expression level & 1 & 20.53 & 20.5297 & 334.79 & $2.2 \times 10^{-16}$ \\
Class & 2 & 2.69 & 1.3467 & 21.96 & $2.98 \times 10^{-10}$ \\
Expression level $\times$ class & 2 & 3.85 & 1.9239 & 31.37 & $2.50 \times 10^{-14}$ \\
Residuals & 17808 & 1092.02 & 0.0613 & & \\
\hline
\end{tabular}

reproductive protein evolution. Here, we discuss these findings in light of previously observed patterns of reproductive protein evolution, and the factors proposed to drive these patterns.

\section{Female Reproductive Proteins Tend to Evolve More Rapidly}

Our evidence for elevated rates of reproductive protein evolution was observed both as a modest but significant increase in mean $\mathrm{dN} / \mathrm{dS}$ across all reproductive loci, and as a significantly larger proportion of loci showing evidence of adaptive divergence between species $(\mathrm{dN} / \mathrm{dS}>1)$. Interestingly, however, this elevated evolution appears to be more clearly associated with proteins that have female- rather than male-specific functions. Indeed, female reproductive loci predominate among our most rapidly evolving loci, both generally and in analyses of tissue-specific loci. Of the tissue-specific loci found to have $\mathrm{dN} / \mathrm{dS}>1$, all are expressed in a female-specific tissue (either style or ovule) (Supplementary Table 16).

This finding is intriguing. Some previous studies have detected accelerated evolution in proteins with specific female-associated functions, for example, egg proteins involved in sperm-egg interactions (e.g., Galindo et al., 2003). Nonetheless, most studies that include both male and female proteins have found mixed evidence for elevated female-biased or female-specific evolution (e.g., Haerty et al., 2007; Wong, 2010; including in plants, e.g., Gossmann et al., 2016), compared to much clearer accelerated evolution in male-specific or male-biased proteins (reviewed in Wong, 2011; Wilburn and Swanson, 2016). Some of this malefemale difference could simply reflect a comparative lack of knowledge about female-associated proteins, leading them to be systematically underexamined in many comparisons. Indeed, most prior studies in animals (reviewed, for example, in Clark et al., 2006; Dapper and Wade, 2020), as well as some in plants (Arunkumar et al., 2013; Harrison et al., 2019; and references therein), have focused largely or entirely on reproductive proteins with male-specific functions. Here, our loci were defined by tissue-specific patterns of gene expression, rather than from a priori expectations of function, which might have helped to ameliorate any such biases.

Our data also indicates a role for adaptive evolution in the observed acceleration of female protein divergence. Elevated rates of non-synonymous change (but values of $\mathrm{dN} / \mathrm{dS}$ still less than 1) could result from relaxed purifying selection (see further below) or because positive selection has only acted on a subset of sites within each gene, or a combination of these effects. However, we also found a larger proportion of female (style and ovule) loci with $\mathrm{dN} / \mathrm{dS}>1$, suggesting a concentration of adaptive female protein evolution specifically involving post-pollination reproductive processes. One driver of this could be sexual interactions. In many animals, where female choice can be exerted both before and after mating (the latter as 'cryptic female choice') (Andersson and Simmons, 2006), however, in angiosperms male-female (pollen-pistil) interactions can only act after pollen arrives at a flower. Therefore, all evolutionary dynamics involving female choice or male-female interactions must act upon loci expressed in this relatively narrow post-mating window. Indeed, among our rapidly evolving $(\mathrm{dN} / \mathrm{dS}>1)$ style loci, we detect at least one protein with putative functions in pollen interactions (a pistil-specific extension-like protein; Solyc02g078080), in addition to several biosynthetic 
enzymes (Supplementary Table 16). (In contrast, $2 / 3$ rapid ovule-specific genes have potential functions in stress response, possibly suggesting roles that are not directly related to sexual interactions-see below). Moreover, in other angiosperms, 'female' loci that show rapid adaptive divergence are also mostly pistil-specific proteins involved in selection among different pollen genotypes (via roles in genetic self-incompatibility) (Clark et al., 2006), again suggesting the importance of post-pollination sexual dynamics in accelerated reproductive protein evolution.

Whether this potential adaptive explanation extends to explain the general elevation of female reproductive protein evolution (Figure 2) or reproductive proteins overall (Figure 1) is not yet clear. One prior study that compared protein evolution in male, female, and non-reproductive tissues in Arabidopsis thaliana and two relatives, also found that mean $\mathrm{dN} / \mathrm{dS}$ was elevated in loci with expression biased toward female tissues (egg cell, central cell, and synergid cells: three haploid tissues within the female gametophyte) but not in pollen-biased proteins (Gossmann et al., 2014). This pattern was attributed to relaxed selection on female-biased loci, partly because additional sitespecific tests did not consistently detect evidence of positive selection in these loci (Gossmann et al., 2014). Because our four species are closely related, we do not have sufficient power to assess site-specific evidence for adaptive evolution in loci whose estimated $\mathrm{dN} / \mathrm{dS}$ is less than 1 . Future analyses that pair divergence-based estimates with polymorphism data within species-that can provide alternative and often more powerful tests of positive and relaxed selection (e.g., Arunkumar et al., 2013; Gossmann et al., 2016) —will be helpful in differentiating the relative influence of these different forms of selection on the broader patterns of elevated $\mathrm{dN} / \mathrm{dS}$ detected here.

Regardless, if elevated female protein evolution is at least partly driven by post-pollination sexual interactions, it is interesting that we do not also observe elevated evolution in pollen-acting proteins. Of prior analyses in angiosperms, several have detected broadly elevated evolution in proteins that are pollen specific (e.g., Arunkumar et al., 2013; Gossmann et al., 2016; Harrison et al., 2019). In principle, pollen protein evolution could be more constrained than style or ovule evolution due to factors like differences in the degree of tissue-specificity or in the operation of haploid selection, but these explanations are not generally consistent with our data (see further below). Alternatively, it's possible that our analysis did not capture all the male proteins relevant to post-pollination sexual interactions. For instance, pollen loci whose expression requires direct interactions with the style and/or other pollen tubes would not be captured in our expression atlas (which was generated from RNA expression in mature ungerminated and in vitro germinated pollen; Supplementary Table 1). There is evidence in other angiosperms that pollen transcriptomes can be actively modified during their transit through the style, resulting in changes in the suite of proteins being expressed (e.g., Qin et al., 2009; Mizukami et al., 2016). Accounting for and including these genes would require transcriptomes from 'mixed' post-pollination tissue, as well as specific approaches to differentiate pollen- from stylarexpression in these mixed samples (e.g., Pease et al., 2016b). Importantly, this limitation would also extend to any style- or ovule-specific proteins that are only elicited during pollination, so may not be a complete explanation for the general difference we detect between male and female loci here.

Other explanations for the under-accounting of pollen loci could include technical effects such as detection limits on gene expression in pollen, and/or exclusion of pollen-expressed loci that are especially short or rapidly evolving, due to filtering during transcript-mapping steps. In addition, by using $\mathrm{dN} / \mathrm{dS}$ comparisons only, we would not have detected protein changes that involve duplicate, chimeric, or novel genes, and/or if different loci are the targets of selection in different lineages. If these latter factors do contribute to our failure to detect elevated pollen protein evolution, it suggests that different dynamics shape pollen protein identity and/or evolution in Solanum compared to other angiosperms that have shown evidence for elevated evolution using metrics like dN/dS (e.g., Arunkumar et al., 2013; Gossmann et al., 2016; Harrison et al., 2019). Regardless, with the data that we currently have, we do not see evidence that male functions are subject to especially intense sexual selection, particularly in comparison to female reproductive proteins.

\section{Rates of Protein Evolution Are Affected by Tissue-Specificity but Not Haploid Expression}

Our results can also suggest factors that shape the patterns of reproductive protein evolution that we do observe, at least among the three broad factors (gene expression level, haploid expression, and/or mating system variation) that we addressed here.

Of these, we find the strongest evidence that rates of protein evolution are influenced by the breadth and magnitude of gene expression. Many studies have shown higher rates of protein evolution in genes with more narrow expression domains and/or lower levels of gene expression (Meisel, 2011; Slotte et al., 2011; and references therein), a relationship that is thought to reflect lower constraint on tissue-specific proteins and stronger purifying selection on genes with high expression levels or broad expression domains. We similarly found that higher rates of protein evolution were associated with greater tissue-limitation and (in some cases) lower levels of gene expression in our species. However, for reproductive protein evolution specifically, we infer that tissue-limitation (a more narrow expression domain) is likely more important than magnitude of gene expression. For the latter, gene expression was not significantly associated with protein evolution for reproductive genes (Supplementary Figure 3 and Supplementary Table 13), and reproductive loci also had much higher mean gene expression levels in comparison to generally expressed genes and, especially, vegetative loci (Supplementary Table 14).

High tissue-specificity has been proposed as an important contributor to elevated rates of reproductive protein evolutionand a better predictor of this than sex-biased gene expression per se-in both animals (Meisel, 2011) and some plants (Veltsos, 2019). For example, in Arabidopsis thaliana and close relatives, Harrison et al. (2019) found faster protein evolution in both pollen-specific and tissue-specific sporophytic genes compared to loci expressed in $>1$ tissue, although pollen-specific loci still 
retain the highest evolutionary rates in this and other analyses in these species (e.g., Szovenyi et al., 2013; Gossmann et al., 2016). Arunkumar et al. (2013) also inferred a role for tissue-limitation or specificity in elevated $\mathrm{dN} / \mathrm{dS}$ in Capsella grandiflora pollen proteins (although this elevation was attributed to different forms of selection-relaxed purifying selection in pollen germ cell genes versus greater purifying and positive selection in pollen tube loci-depending on the specific sub-tissues within pollen). These prior inferences, and our observations, suggest that higher tissue-specificity could facilitate broadly elevated rates of protein evolution. Nonetheless, tissue-specificity cannot provide a complete explanation of elevated reproductive protein evolution in our dataset, as tissue-specific non-reproductive proteins still evolve more slowly than tissue-specific (female) reproductive proteins (Table 2 ).

In comparison, we found no rate differences between loci expressed in haploid versus diploid tissues, and therefore little evidence for a strong effect of haploid selection in driving differences between reproductive and non-reproductive proteins. Haploid selection has two projected effects on haploid-expressed loci-increasing the efficacy of positive selection on advantageous alleles, and of purifying selection on deleterious alleles (Immler and Otto, 2018; Mattila et al., 2018)-compared to diploidexpressed loci. We have limited ability to differentiate these two effects with our particular estimate of protein evolution, but our observations are not consistent with a strong effect of either. In terms of accelerating adaptive protein evolution, we found no significant elevation of evolutionary rates in loci limited to gametophytic-tissues in general, and in ovule-limited and pollen-limited loci specifically (Table 3 ). Similarly, we find little evidence for stronger or more widespread purifying selection on haploid-expressed loci, which would be observed as lower mean rates of protein evolution in gametophytic loci. Stronger purifying selection due to haploid expression has been proposed as one reason why sex-biased loci (generally, pollen expressed loci) do not appear to evolve faster than non-sex-biased loci in several dioecious species (Cossard et al., 2019; Sanderson et al., 2019; discussed in Muyle, 2019; but see also Veltsos, 2019) and, indeed, sometimes appear to evolve more slowly (e.g., Darolti et al., 2018). Our data do not suggest stronger purifying selection in either gametophytic-limited proteins in general, or ovule- and pollen-limited loci specifically, in our species. However, because we have relatively few pollen-specific loci (discussed above) we cannot exclude the possibility that intermediate rates of pollen protein evolution result from a tension between both positive and purifying selection predicted to act on these loci. Nonetheless, our present data indicates little evidence for a global effect of haploid gene expression on molecular evolutionary rates.

\section{Mating System Variation Does Not Differentially Influence Reproductive Protein Evolution}

Our results also did not support a strong consistent effect of mating system differences-and therefore predicted differences in the strength of sexual selection-on patterns of reproductive protein evolution. Interpreting these comparisons is complex because differences in genetic effective population size are expected to have global effects on rates of molecular evolution (Charlesworth and Wright, 2001; and see the section "Introduction") that are unrelated to sexual selection per se. The broad patterns of $\mathrm{dN} / \mathrm{dS}$ we observed generally reflected these expected global effects of differences in Ne due to mating system variation: in the SC lineages, mean $\mathrm{dN} / \mathrm{dS}$ was higher in both RP and GR genes, consistent with a general relaxation of selection in more selfing lineages (Charlesworth and Wright, 2001) regardless of whether or not loci were associated with reproductive functions (Table 4). This genome-wide effect might have overwhelmed more subtle effects of mating system variation acting specifically on loci involved in male-male competition and/or female choice. In addition, the transition to self-compatibility is relatively recent (less than $1 \mathrm{MY}$ ) among our species, and self-compatible lineages still retain significant potential for outcrossing (Rick, 1979; Vosters et al., 2014), which generally ranges from 0 to $40 \%$ (e.g., Rick et al., 1977) but has been estimated as high as $84 \%$ in some genotypes (Rick et al., 1978). These factors mean there may be only modest differences in the historical and current opportunity for sexual selection among our lineages, and therefore limited opportunity to observe systematic consequences of this transition as differential changes in evolutionary rates, especially among a sample of four species. (Statistically, this is compounded in our particular dataset by the two SC species branches being substantially shorter than those for our two SI species). This contrasts with some other angiosperms, such as A. thaliana, where the transition to self-compatibility is about as old but the consequent effects on outcrossing rates has been much more severe (outcrossing rates are frequently $<5 \%$; Harrison et al., 2019; and references therein). Even among Arabidopsis, however, the effects of mating system on sexual locus evolution might be complex. For example, Gossmann et al. (2014) found that $\mathrm{dN} / \mathrm{dS}$ in pollen-acting genes was significantly lower in the outcrosser A. lyrata compared to selfing $A$. thaliana, even though pollen competition could be expected to accelerate adaptive evolution in these loci in the outbreeding species.

Interestingly, several previous analyses in animal systems have similarly found equivocal evidence for differential effects of mating system variation on the evolution of reproductive tract proteins (reviewed in Wong, 2011). For example, in studies of primates, evolutionary rates in specific sperm and copulatory plug proteins are positively associated with common proxies for sexual selection, such as residual testis size and estimated number of mates (e.g., Dorus et al., 2004; Martin-Coello et al., 2009). However other studies find that phylogeny-wide signals of positive selection in reproductive tract proteins are not associated specifically with polyandrous (as opposed to monogamous) lineages (e.g., Ramm et al., 2008; Finn and Civetta, 2010; and see Table 1 in Wong, 2011). Limitations on phylogenetic models that can assess associations between molecular evolutionary rates and changes in phenotypic characters, and variation among lineages in the specific targets of sexual selection (Wong, 2011), might explain relatively weak effects of mating system variation on 
protein evolution rates, despite the importance of sexual selection in driving faster reproductive protein evolution. Adding more species, and focusing on pairs of SI-SC sister species, might help in addressing some of these limitations in future analyses within Solanum.

Alternatively, the weak effect of mating system variationboth here and in prior studies-might be because the predominant cause of globally elevated reproductive protein evolution is not sexual selection. For example, pathogen resistance responses are also known to be associated with elevated rates of protein evolution-driven by antagonistic host-pathogen evolution-and might be concentrated among reproductive proteins if mating often leads to the exchange of pathogens (Clark et al., 2006; Wong, 2011). In principle, the identity of loci inferred to be under positive selection could be helpful in differentiating among alternative selective agents responsible for driving this adaptive divergence. Of the genes in our dataset that show elevated evolution, we cannot yet draw strong conclusions about whether they better reflect roles related to sexual interactions versus responses to non-sexual environmental factors; many of them have unknown functions or general functions that do not a priori provide specific support for either inference (Supplementary Table 16). We do detect at least one stylespecific protein with a clear functional connection to pollen-pistil interactions. However, our rapidly evolving ovule-specific genes are implicated in stress responses, which might indicate a greater role for natural rather than sexual selection in shaping adaptive responses in this class of loci.

\section{Consequences of Rapid Protein Evolution for the Evolution of Reproductive Differences and Speciation}

Finally, among the motivations for examining patterns of reproductive protein evolution is to understand their possible role in lineage diversification. If reproductive proteins do indeed evolve more rapidly than other classes of protein, then perhaps they play an outsized role in the emergence of functional differences and reproductive isolating barriers between lineages. For example, evidence suggests post-mating prezygotic traits frequently evolve rapidly in animals (e.g., Galindo et al., 2003; Dorus et al., 2004; Plakke et al., 2019) and, when divergent between species, could contribute to reproductive isolation (Howard, 1999; Palumbi, 2009; Snook et al., 2009; Castillo and Moyle, 2014). Similarly, divergence in post-pollination prezygotic reproductive molecules could be important contributors to species barrier formation among angiosperms (Moyle et al., 2014). Here, we did detect evidence for a global elevation of protein evolution in tissues that mediate these post-pollination prezygotic stages, especially of femalespecific loci. Our observations are consistent with a role for tissue

\section{REFERENCES}

Andersson, M., and Simmons, L. W. (2006). Sexual selection and mate choice. Trends Ecol. Evol. 21, 296-302. specificity in facilitating these elevated rates, but not for haploid selection or, as yet, for differences in selective dynamics that are associated with recent shifts in mating system. The evolutionary factors that might drive this more rapid evolution therefore remain to be clarified. Regardless, our results indicate that rapid reproductive divergence is especially characteristic of proteins with female-specific functions among our species. These specific functions can be critical for maintaining coordinated sexual signals and ensuring fertilization post-pollination (Bernasconi et al., 2004; Swanson et al., 2004; Moyle et al., 2014). Therefore their rapid divergence might be particularly influential in the initial stages of reproductive isolation and, thereby, in speciation, in this highly diverse plant genus.

\section{DATA AVAILABILITY STATEMENT}

The datasets presented in this study can be found in online repositories. The names of the repository/repositories and accession number(s) can be found in the article/Supplementary Material.

\section{AUTHOR CONTRIBUTIONS}

LM, MW, and MG designed the study. MW and MG performed the analyses. LM wrote the manuscript with assistance from MW and MG. All authors contributed to the article and approved the submitted version.

\section{FUNDING}

This work was supported by NSF grant IOS-1127059 and NSF grant DEB-1856469 to LM.

\section{ACKNOWLEDGMENTS}

Thanks to C. J. Jewell for collecting ovule tissue, Christie Bergeron for RNA extractions, the Indiana University Center for Genomics and Bioinformatics for assistance with library construction and sequencing, and the reviewers for comments that helped improve the study.

\section{SUPPLEMENTARY MATERIAL}

The Supplementary Material for this article can be found online at: https://www.frontiersin.org/articles/10.3389/fpls.2021. 635990/full\#supplementary-material

Andersson, M. B. (1994). Sexual Selection. Princeton, NJ: Princeton University Press.

Arunkumar, R., Josephs, E. B., Williamson, R. J., and Wright, S. I. (2013). Pollenspecific, but not sperm-specific, genes show stronger purifying selection and 
higher rates of positive selection than sporophytic genes in Capsella grandiflora. Mol. Biol. Evol. 30, 2475-2486. doi: 10.1093/molbev/mst149

Ashburner, M., Ball, C. A., Blake, J. A., Botstein, D., Butler, H., Cherry, J. M., et al. (2000). Gene Ontology: tool for the unification of biology. Nat. Genet. 25, 25-29.

Bernasconi, G., Ashman, T. L., Birkhead, T. R., Bishop, J. D. D., Grossniklaus, U., Kubli, E., et al. (2004). Evolutionary ecology of the prezygotic stage. Science 303, 971-975. doi: 10.1126/science. 1092180

Bolger, A. M., Lohse, M., and Usadel, B. (2014). Trimmomatic: a flexible trimmer for Illumina sequence data. Bioinformatics 30, 2114-2120. doi: 10.1093/ bioinformatics/btu170

Brandvain, Y., and Haig, D. (2005). Divergent mating systems and parental conflict as a barrier to hybridization in flowering plants. Am. Natural. 166, 330-338. doi: $10.2307 / 3473312$

Castillo, D. M., and Moyle, L. C. (2014). Intraspecific sperm competition genes enforce post-mating species barriers in Drosophila. Proc. R. Soc. B-Biol. Sci. 281:1797.

Charlesworth, B., Morgan, M. T., and Charlesworth, D. (1993). The effect of deleterious mutations on neutral molecular variation. Genetics. 134, 1289-1303. doi: 10.1093/genetics/134.4.1289

Charlesworth, D., and Charlesworth, B. (1992). The effects of selection in the gametophytic stage on mutational load. Evolution. 46, 703-720. doi: 10.2307/ 2409639

Charlesworth, D., and Wright, S. I. (2001). Breeding systems and genome evolution. Curr. Opin. Genet. Dev. 11, 685-690. doi: 10.1016/s0959-437x(00) 00254-9

Clark, N. L., Aagaard, J. E., and Swanson, W. J. (2006). Evolution of reproductive proteins from animals and plants. Reproduction 131, 11-22. doi: 10.1530/rep.1. 00357

Cossard, G. G., Toups, M. A., and Pannell, J. R. (2019). Sexual dimorphism and rapid turnover in gene expression in pre-reproductive seedlings of a dioecious herb. Ann. Bot. 123, 1119-1131. doi: 10.1093/aob/mcy183

Cruz-Garcia, F., Hancock, C. N., and Mcclure, B. (2003). S-RNase complexes and pollen rejection. J. Exp. Bot. 54, 123-130. doi: 10.1093/jxb/erg045

Dapper, A. L., and Wade, M. J. (2020). Relaxed Selection and the Rapid Evolution of Reproductive Genes. Trends Genet. 36, 640-649. doi: 10.1016/j.tig.2020.06.014

Darolti, I., Wright, A. E., Pucholt, P., Berlin, S., and Mank, J. E. (2018). Slow evolution of sex-biased genes in the reproductive tissue of the dioecious plant Salix viminalis. Mole. Ecol. 27, 694-708. doi: 10.1111/mec.14466

Delph, L. F., and Ashman, T. L. (2006). Trait selection in flowering plants: how does sexual selection contribute? Int. Comparat. Biol. 46, 465-472. doi: 10.1093/icb/ icj038

Delph, L. F., and Havens, K. (1998). "Pollen competition in flowering plants," in Sperm competition and sexual selection, eds B. TR and M. AP (San Diego, CA: Academic Press), 149-173. doi: 10.1016/b978-012100543-6/50030-1

Dobin, A., Davis, C. A., Schlesinger, F., Drenkow, J., Zaleski, C., Jha, S., et al. (2013). STAR: ultrafast universal RNA-seq aligner. Bioinformatics 29, 15-21. doi: 10.1093/bioinformatics/bts635

Dorus, S., Evans, P. D., Wyckoff, G. J., Choi, S. S., and Lahn, B. T. (2004). Rate of molecular evolution of the seminal protein gene SEMG2 correlates with levels of female promiscuity. Nat. Genet. 36, 1326-1329. doi: 10.1038/ ng1471

Finn, S., and Civetta, A. (2010). Sexual Selection and the Molecular Evolution of ADAM Proteins. J. Mole. Evol. 71, 231-240. doi: 10.1007/s00239-0109382-7

Galindo, B. E., Vacquier, V. D., and Swanson, W. J. (2003). Positive selection in the egg receptor for abalone sperm lysin. Proc. Natl. Acad. Sci. U S A. 100, 4639-4643. doi: 10.1073/pnas.0830022100

Goldberg, E. E., Kohn, J. R., Lande, R., Robertson, K. A., Smith, S. A., and Igic, B. (2010). Species selection maintains self-incompatibility. Science 330, 493-495. doi: $10.1126 /$ science. 1194513

Gossmann, T. I., Saleh, D., Schmid, M. W., Spence, M. A., and Schmid, K. J. (2016). Transcriptomes of Plant Gametophytes Have a Higher Proportion of Rapidly Evolving and Young Genes than Sporophytes. Mole. Biol. Evol. 33, 1669-1678. doi: $10.1093 / \mathrm{molbev} / \mathrm{msw} 044$

Gossmann, T. I., Schmid, M. W., Grossniklaus, U., and Schmid, K. J. (2014). Selection-Driven Evolution of Sex-Biased Genes Is Consistent with Sexual
Selection in Arabidopsis thaliana. Mole. Biol. Evol. 31, 574-583. doi: 10.1093/ molbev/mst226

Haerty, W., Jagadeeshan, S., Kulathinal, R. J., Wong, A., Ram, K. R., Sirot, L. K., et al. (2007). Evolution in the fast lane: Rapidly evolving sex-related genes in drosophila. Genetics 177, 1321-1335. doi: 10.1534/genetics.107.078865

Hafidh, S., Fila, J., and Honys, D. (2016). Male gametophyte development and function in angiosperms: a general concept. Plant Reproduct. 29, 31-51. doi: 10.1007/s00497-015-0272-4

Hahn, M. W. (2018). Molecular Population Genetics. Sinauer/. Oxford UK: Oxford University Press.

Harrison, M. C., Mallon, E. B., Twell, D., and Hammond, R. L. (2019). Deleterious Mutation Accumulation in Arabidopsis thaliana Pollen Genes: A Role for a Recent Relaxation of Selection. Genome Biol. Evol. 11, 1939-1951. doi: 10.1093/ gbe/evz127

Hothorn, T., Bretz, F., and Westfall, P. (2008). Simultaneous inference in general parametric models. Biomet. J. 50, 346-363. doi: 10.1002/bimj.200810425

Howard, D. J. (1999). Conspecific sperm and pollen precedence and speciation. Ann. Rev. Ecol. Syst. 30, 109-132. doi: 10.1146/annurev.ecolsys.30.1.109

Immler, S. (2019). Haploid Selection in "Diploid" Organisms. Ann. Rev. Ecol. Evol. Systemat. 50, 219-236. doi: 10.1146/annurev-ecolsys-110218-024709

Immler, S., and Otto, S. P. (2018). The evolutionary consequences of selection at the haploid gametic stage. Am. Natural. 192, 241-249. doi: 10.1086/ 698483

Ichihashi, Y., Aguilar-Martinez, J. A., Farhi, M., Chitwood, D. H., Kumar, R., Millon, L. V., et al. (2014). Evolutionary developmental transcriptomics reveals a gene network module regulating interspecific diversity in plant leaf shape. Proc. Natl. Acad. Sci. U S A. 111, E2616-E2621.

Koenig, D., Jimenez-Gomez, J. M., Kimura, S., Fulop, D., Chitwood, D. H., Headland, L. R., et al. (2013). Comparative transcriptomics reveals patterns of selection in domesticated and wild tomato. Proc. Natl. Acad. Sci. U S A. 110, E2655-E2662.

Liao, Y., Smyth, G. K., and Shi, W. (2014). featureCounts: an efficient general purpose program for assigning sequence reads to genomic features. Bioinformatics 30, 923-930. doi: 10.1093/bioinformatics/btt656

Lloyd, D. G., and Webb, C. J. (1977). Secondary sexual characters in plants. Botanical Review. 43, 177-216.

Martin-Coello, J., Dopazo, H., Arbiza, L., Ausio, J., Roldan, E. R. S., and Gomendio, M. (2009). Sexual selection drives weak positive selection in protamine genes and high promoter divergence, enhancing sperm competitiveness. Proc. R. Soc. B-Biol. Sci. 276, 2427-2436. doi: 10.1098/rspb.2009.0257

Mattila, T. M., Laenen, B., and Slotte, T. (2018). Population genomics of transitions to selfing in Brassicaceae model systems. Arxiv doi: 1809.04872

Mazer, S. J., Hendrickson, B. T., Chellew, J. P., Kim, L. J., Liu, J. W., Shu, J., et al. (2018). Divergence in pollen performance between Clarkia sister species with contrasting mating systems supports predictions of sexual selection. Evolution 72, 453-472. doi: 10.1111/evo.13429

Mazer, S. J., Hove, A. A., Miller, B. S., and Barbet-Massin, M. (2010). The joint evolution of mating system and pollen performance: Predictions regarding male gametophytic evolution in selfers vs. outcrossers. Perspect. Plant Ecol. Evolut. Syst. 12, 31-41. doi: 10.1016/j.ppees.2009.06.005

Mcclure, B., Cruz-Garcia, F., and Romero, C. (2011). Compatibility and incompatibility in S-RNase-based systems. Ann. Bot. 108, 647-658. doi: 10. 1093/aob/mcr179

Meisel, R. P. (2011). Towards a More Nuanced Understanding of the Relationship between Sex-Biased Gene Expression and Rates of Protein-Coding Sequence Evolution. Mole. Biol. Evol. 28, 1893-1900. doi: 10.1093/molbev/msr010

Mi, H., Huang, X., Muruganujan, A., Tang, H., Mills, C., Kang, D., et al. (2019). PANTHER version 14: more genomes, a new PANTHER GO-slim and improvements in enrichment analysis tools. Nucleic Acids Res. 47, D419-D426.

Mizukami, A. G., Inatsugi, R., Jiao, J., Kotake, T., Kuwata, K., Ootani, K., et al. (2016). The AMOR Arabinogalactan Sugar Chain Induces Pollen-Tube Competency to Respond to Ovular Guidance. Curr. Biol. 26, 1091-1097. doi: 10.1016/j.cub.2016.02.040

Mizuta, Y., and Higashiyama, T. (2018). Chemical signaling for pollen tube guidance at a glance. J. Cell Sci. 131:jcs208447. doi: 10.1242/jcs.208447

Moore, J. C., and Pannell, J. R. (2011). Sexual selection in plants. Curr. Biol. 21, R176-R182. 
Moyle, L. C., Jewell, C. P., and Kostyun, J. L. (2014). Fertile approaches to dissecting mechanisms of premating and postmating prezygotic reproductive isolation. Curr. Opin. Plant Biol. 18, 16-23. doi: 10.1016/j.pbi.2013.12.005

Muyle, A. (2019). How different is the evolution of sex-biased gene expression between plants and animals? A commentary on: 'Sexual dimorphism and rapid turnover in gene expression in prereproductive seedlings of a dioecious herb'. Ann. Bot. 123, IV-V.

Palumbi, S. R. (2009). Speciation and the evolution of gamete recognition genes: pattern and process. Heredity 102: 66-76. doi: 10.1038/hdy.2008.104

Pease, J. B., Haak, D. C., Hahn, M. W., and Moyle, L. C. (2016a). Phylogenomics reveals three sources of adaptive variation during a rapid radiation. PLoS Biol. 14, e1002379. doi: 10.1371/journal.pbio.1002379

Pease, J. B., Guerrero, R. F., Sherman, N. A., Hahn, M. W., and Moyle, L. C. (2016b). Molecular mechanisms of postmating prezygotic reproductive isolation uncovered by transcriptome analysis. Mole. Ecol. 25, 2592-2608. doi: 10.1111/mec.13679

Peters, M. A. E., and Weis, A. E. (2018). Selection for pollen competitive ability in mixed-mating systems. Evolution. 72, 2513-2536. doi: 10.1111/evo.13597

Plakke, M. S., Walker, J. L., Lombardo, J. B., Goetz, B. J., Paceila, G. N., and Durrant, J. D. (2019). Characterization of female reproductive proteases in a butterfly from functional and evolutionary perspectives. Physiolog.Biochem. Zool. 92, 579-590. doi: 10.1086/705722

Qin, Y., Leydon, A. R., Manziello, A., Pandey, R., Mount, D., Denic, S., et al. (2009). Penetration of the Stigma and Style Elicits a Novel Transcriptome in Pollen Tubes, Pointing to Genes Critical for Growth in a Pistil. PLoS Genet. 5:e1000621. doi: 10.1371/journal.pgen.1000621

R Core Team (2015). R: A language and environment for statistical computing. Vienna, Austria: Sponsored by the R Foundation for Statistical Computing.

Ramm, S. A., Oliver, P. L., Ponting, C. P., Stockley, P., and Emes, R. D. (2008). Sexual selection and the adaptive evolution of mammalian ejaculate proteins. Mole. Biol. Evol. 25, 207-219. doi: 10.1093/molbev/msm 242

Rick, C. M. (1979). "Biosystematic studies in Lycopersicon and closely related species of Solanum," in The biology and taxonomy of the Solanaceae, eds J. G. Hawkes, R. N. Lester, and A. D. Skelding (New York,NY: Academic Press Inc), 667-679.

Rick, C. M., and Fobes, J. F. (1975). Allozyme variation in cultivated tomato and closely related species. Bull. Torrey Botanic. Club 102, 376-384. doi: 10.2307/ 2484764

Rick, C. M., Fobes, J. F., and Holle, M. (1977). Genetic variation in Lycopersicon pimpinellifolium - evidence of evolutionary change in mating systems. Plant Systemat. Evol. 127, 139-170. doi: 10.1007/bf00984147

Rick, C. M., Holle, M., and Thorp, R. W. (1978). Rates of cross-pollination in Lycopersicon pimpinellifolium - impact of genetic variation in floral characters. Plant Syst. Evol. 129, 31-44. doi: 10.1007/bf00988982

Ritchie, M. G. (2007). Sexual selection and speciation. Ann. Rev. Ecol. Evol. Syst. 38, 79-102.

Sanderson, B. J., Wang, L., Tiffin, P., Wu, Z. Q., and Olson, M. S. (2019). Sex-biased gene expression in flowers, but not leaves, reveals secondary sexual dimorphism in Populus balsamifera. N. Phytol. 221, 527-539. doi: 10.1111/nph.15421

Sarkinen, T., Bohs, L., Olmstead, R. G., and Knapp, S. (2013). A phylogenetic framework for evolutionary study of the nightshades (Solanaceae): a dated 1000-tip tree. Bmc Evol. Biol. 2013:13.

Sicard, A., and Lenhard, M. (2011). The selfing syndrome: a model for studying the genetic and evolutionary basis of morphological adaptation in plants. Ann. Bot. 107, 1433-1443. doi: 10.1093/aob/mcr023

Skogsmyr, I., and Lankinen, A. (2002). Sexual selection: an evolutionary force in plants. Biol. Rev. 77, 537-562. doi: 10.1017/s1464793102005973

Slotte, T., Bataillon, T., Hansen, T. T., Stonge, K. R., Wright, S. I., and Schierup, M. H. (2011). Genomic Determinants of Protein Evolution and Polymorphism in Arabidopsis. Genome Biol. Evol. 3, 1210-1219. doi: 10.1093/gbe/evr094

Snook, R. R., Chapman, T., Moore, P. J., Wedell, N., and Crudgington, H. S. (2009). Interactions between the sexes: new perspectives on sexual selection and reproductive isolation. Evol. Ecol. 23, 71-91. doi: 10.1007/s10682-0079215-3

Stephenson, A. G., and Bertin, R. I. (1983). Male competition, female choice, and sexual selection in plants. In: Pollination Biology. New York, NY: Academic Press, 109-151.

Stone, J. L. (2002). Molecular mechanisms underlying the breakdown of gametophytic self-incompatibility. Q. Rev. Biol. 77, 17-32. doi: 10.1086/339200

Swanson, R., Edlund, A. F., and Preuss, D. (2004). Species specificity in pollenpistil interactions. Ann. Rev. Genet. 38, 793-818. doi: 10.1146/annurev.genet. 38.072902.092356

Swanson, W. J., and Vacquier, V. D. (2002a). The rapid evolution of reproductive proteins. Nat. Rev. Genet. 3, 137-144. doi: 10.1038/nrg733

Swanson, W. J., and Vacquier, V. D. (2002b). Reproductive protein evolution. Ann. Rev. Ecol. Syst. 33, 161-179. doi: 10.1146/annurev.ecolsys.33.010802.150439

Szovenyi, P., Ricca, M., Hock, Z., Shaw, J. A., Shimizu, K. K., and Wagner, A. (2013). Selection Is No More Efficient in Haploid than in Diploid Life Stages of an Angiosperm and a Moss. Mole. Biol. Evol. 30, 1929-1939. doi: 10.1093/ molbev/mst095

Takayama, S., and Isogai, A. (2005). Self-incompatibility in plants. Annu. Rev. Plant Biol. 56, 467-489.

The Gene Ontology Consortium. (2019). The Gene Ontology Resource: 20 years and still GOing strong. Nucleic Acids Res. 47, D330-D338.

Turner, L. M., and Hoekstra, H. E. (2008). Causes and consequences of the evolution of reproductive proteins. Int. J. Dev. Biol. 52, 769-780. doi: 10.1387/ ijdb.082577lt

Vacquier, V. D., and Swanson, W. J. (2011). Selection in the Rapid Evolution of Gamete Recognition Proteins in Marine Invertebrates. Cold Spring Harb. Perspect. Biol. 3:a002931. doi: 10.1101/cshperspect.a002931

Veltsos, P. (2019). Not all sex-biased genes are the same. N. Phytol. 221, 10-11. doi: $10.1111 /$ nph.15531

Vosters, S. L., Jewell, C. P., Sherman, N. A., Einterz, F., Blackman, B. K., and Moyle, L. C. (2014). The timing of molecular and morphological changes underlying reproductive transitions in wild tomatoes (Solanum sect. Lycopersicon). Mole. Ecol. 23, 1965-1978. doi: 10.1111/mec.12708

Walsh, N. E., and Charlesworth, D. (1992). Evolutionary interpretations of differences in pollen-tube growth rates. Q. Rev. Biol. 67, 19-37. doi: 10.1086/ 417446

Wilburn, D. B., and Swanson, W. J. (2016). From molecules to mating: Rapid evolution and biochemical studies of reproductive proteins. J. Proteom. 135, 12-25. doi: 10.1016/j.jprot.2015.06.007

Willson, M. F. (1979). Sexual selection in plants. Am. Natural. 113, 777-790.

Willson, M. F. (1994). Sexual selection in plants - perspective and overview. Am. Natural. 144, S13-S39.

Wong, A. (2010). Testing the effects of mating system variation on rates of molecular evolution in primates. Evolution 64, 2779-2785. doi: 10.1111/j.15585646.2010.01038.x

Wong, A. (2011). The molecular evolution of animal reproductive tract proteins: what have we learned from mating-system comparisons? Int. J. Evol. Biol. 2011, 1-9. doi: 10.4061/2011/908735

Yang, Z. (2007). PAML 4: Phylogenetic Analysis by Maximum Likelihood. Mole. Biol. Evol. 24, 1586-1591. doi: 10.1093/molbev/msm088

Conflict of Interest: The authors declare that the research was conducted in the absence of any commercial or financial relationships that could be construed as a potential conflict of interest.

Copyright (C) 2021 Moyle, Wu and Gibson. This is an open-access article distributed under the terms of the Creative Commons Attribution License (CC BY). The use, distribution or reproduction in other forums is permitted, provided the original author(s) and the copyright owner(s) are credited and that the original publication in this journal is cited, in accordance with accepted academic practice. No use, distribution or reproduction is permitted which does not comply with these terms. 